\title{
GMR
}

\section{Exceptional material requirement for reproduction in mouse oocytes}

\author{
L. Yu ${ }^{1 *}$, S.F. Wang ${ }^{2 *}$, Q.Z. Zhai ${ }^{3 *}$, Y.Q. Yao ${ }^{3}$, F. Jiang ${ }^{4}$ and Y.X. Lu ${ }^{1}$ \\ ${ }^{1}$ Department of Obstetrics and Gynecology, \\ First Affiliated Hospital of the General Hospital of the People's Liberation Army, \\ Beijing, China \\ ${ }^{2}$ Blood Transfusion Department, \\ The General Hospital of the People's Liberation Army, Beijing, China \\ ${ }^{3}$ Department of Obstetrics and Gynecology, \\ The General Hospital of the People's Liberation Army, Beijing, China \\ ${ }^{4}$ Department of Obstetrics and Gynecology, Tangdu Hospital, \\ Fourth Military Medical University, Xi'an, China \\ ${ }^{*}$ These authors contributed equally to this study. \\ Corresponding author: Y.X. Lu \\ E-mail: yongxianlu2014@126.com
}

Genet. Mol. Res. 14 (4): 14356-14365 (2015)

Received May 13, 2015

Accepted August 21, 2015

Published November 13, 2015

DOI http://dx.doi.org/10.4238/2015.November.13.21

ABSTRACT. Limited information on oocytes and fertilization prevents the efficient therapy of patients with infertility. The most important reason for this lack of understanding is a deficiency in research dedicated to oocytes and fertilization. Currently, we are concerned with the role of nutrition in the process of oocyte development to better understand the relationship between nutrition and infertility. The aim of this study was to explore the relationship between some exceptional materials and infertility to elucidate the role of these materials in oocyte development. We used proteomic analysis to identify numerous nutrition-related proteins in three developmental stages: the germinal vesicle stage, the metaphase II-arrested stage, and the fertilized oocyte-zygote stage. Specific proteins were abundantly expressed during the three stages. 
These proteins included astacin-like metalloendopeptidase, selenium-binding proteins, and other proteins involved in metabolic and signaling pathways. Other proteins were involved in the citrate cycle, the electron transport chain, the urea cycle, fatty acid metabolism, and the insulin signaling pathway. Almost all these proteins exhibited different expression levels in the three stages. The results of the present study provide a better understanding of the molecular mechanisms of early embryonic development and suggest new treatment methods for infertility.

Key words: Oocyte; Zygote; Protein; Nutrition; Fertilization

\section{INTRODUCTION}

Infertility is defined as not having conceived after months of contraceptive-free intercourse in a female under the age of 34 . Infertility is a worldwide problem that affects $9-15 \%$ of the childbearing population, and $55 \%$ of those affected seek medical advice in the hope of achieving parenthood (Gurunath et al., 2011). The fertilization process of oocytes appears to be simple. However, multiple signaling pathways are involved in the process, and many factors including minerals, some specific proteins, and many nutrients can regulate these pathways. Unfortunately, little is known about the oocyte fertilization process. For example, many embryos develop well but cannot achieve implantation, and many oocytes appear viable but cannot be fertilized. From the time of fertilization, embryos need abundant nutrition, but exactly which nutrients are important is unknown. Additionally, with the increasing popularity of in vitro fertilization (IVF) methods in recent years, a deeper understanding of the molecular composition of oocytes may be helpful in increasing the success rates of IVF.

A comparison of the proteomes of oocytes at different developmental stages may aid in the identification of important factors responsible for fertilization and implantation. To date, most functional analyses of oocyte proteins have been limited to proteins that are primarily expressed in the oocyte. However, functional analysis of abundant non-oocyte-restricted proteins in a developmental context would also be highly informative. In our study, proteomes of mouse oocytes at various developmental stages were analyzed. The study identifies numerous proteins that have not been previously reported, and some proteins were abundantly expressed in the oocytes at different developmental stages. These proteins may provide an important source of nutrition for developing oocytes.

\section{MATERIAL AND METHODS}

\section{Collection of mouse oocytes and zygotes}

The specific-pathogen-free-grade hybrid mice B6D2F1 (C57BL/6 x DBA/2) were housed in the animal facility of the National Institute of Biological Sciences. All studies adhered to procedures consistent with the National Institute of Biological Sciences Guide for the care and use of laboratory animals. Fully grown germinal vesicle (GV) oocytes were collected from 4-8-week-old B6D2F1 female mice 44-48 h after pregnant mare serum gonadotropin (PMSG) injection. The antral follicles were punctured by 30-gauge needles, and GV oocytes enclosed by several layers of cumulus cells were selected. The cumulus cells were mechanically removed, and the denuded oocytes were 
collected. Metaphase II-arrested (MII) oocytes were collected from the oviducts of B6D2F1 mice after sequential injection of 8-12-week-old females with PMSG and human chorionic gonadotropin (hCG). Adherent cumulus cells were removed by hyaluronidase treatment, and the cumulus cellfree MII oocytes were collected. The zygotes were collected from the oviducts of female B6D2F1 mice that had been mated with male mice.

\section{Protein extraction}

Oocyte proteins were extracted using FastPrep-24 (MP Biomedicals, Santa Ana, California, USA). Briefly, all samples were resuspended with $50 \mu \mathrm{L}$ phosphate-buffered saline. Next, $170 \mu \mathrm{L}$ lysis buffer (20 mM Hepes, pH 7.4, $10 \mathrm{mM} \mathrm{KCl}, 1.5 \mathrm{mM} \mathrm{MgCl}, 1 \mathrm{mM}$ dithiothreitol) and $7 \mu \mathrm{L} 50$ x CPI (complete, ethylenediaminetetraacetic acid-free; Roche) was added and transferred to a 1.5-mL screwcapped tube containing $300 \mu \mathrm{L}$ pre-chilled $0.5-\mathrm{mm}$-diameter glass beads. The oocytes were lysed twice using a FastPrep-24 instrument set to speed 6 for $20 \mathrm{~s}$, and the samples were chilled on ice for 3 min between spins. The lysates were collected in a 2-mL tube by piercing a hole at the bottom of the 1.5-mL screw-capped tube and spinning at $300 \times g$ for $1 \mathrm{~min}$. Next, the lysates were centrifuged at 13,400 $\mathrm{xg}$ for $20 \mathrm{~min}$. Proteins in the supernatants were precipitated with $25 \%$ trichloroacetic acid (TCA) for 30 min on ice and centrifuged at 13,400 x $g$ for $30 \mathrm{~min}$. The pellets from TCA precipitation were washed twice with cold acetone, air-dried, and dissolved in $70 \mu \mathrm{L} 100 \mathrm{mM}$ Tris $(\mathrm{pH} 8.5)$ and $8 \mathrm{M}$ urea.

\section{Digestion}

The lysate supernatant protein solutions were reduced with $5 \mathrm{mM}$ tris-(2-carboxyethyl)phosphine (Pierce) at room temperature for $20 \mathrm{~min}$ and then alkylated with $10 \mathrm{mM}$ iodoacetamide at room temperature for $20 \mathrm{~min}$. After a four-fold dilution with $100 \mathrm{mM}$ Tris ( $\mathrm{pH} \mathrm{8.5),} \mathrm{methylamine}$ was added to a concentration of $20 \mathrm{mM}$ to reduce carbamylation, and $\mathrm{CaCl}_{2}$ was added to a concentration of $1 \mathrm{mM}$. The samples were digested with trypsin (substrate-to-enzyme mass-tomass ratio of $50: 1$ ) at $37^{\circ} \mathrm{C}$ for $16 \mathrm{~h}$. The reactions were quenched with the addition of formic acid to a concentration of $5 \%$, and insoluble particulates were removed by centrifugation $(14,000 \times \mathrm{g}$ for $20 \mathrm{~min}$ ). Digested supernatant fractions were stored at $-80^{\circ} \mathrm{C}$ without further treatment until required for mass spectrometer (MS) analysis. The pellet fractions of the oocyte lysates were resuspended in $10 \mu \mathrm{L} 5 \mathrm{x}$ Invitrosol liquid chromatography-mass spectrometry (LC-MS) surfactant blend, vortexed, and incubated for $5 \mathrm{~min}$ at $60^{\circ} \mathrm{C}$. Both samples were diluted with $10 \mu \mathrm{L} \mathrm{H} \mathrm{H}_{2}, 64 \mu \mathrm{L}$ $100 \%$ acetonitrile, and $16 \mu \mathrm{L} 500 \mathrm{mM}$ Tris ( $\mathrm{pH} 8.5)$, and then sealed with parafilm. After 20-30 min sonication, the samples were digested with trypsin (substrate-to-enzyme mass-to-mass ratio of 50: 1) at $37^{\circ} \mathrm{C}$ for $16 \mathrm{~h}$. The reactions were quenched with the addition of formic acid to a concentration of $5 \%$, and insoluble particulates were removed by centrifugation (14,000 x $g$ for $20 \mathrm{~min}$ ). The solutions were reduced to less than $50 \mu \mathrm{L}$ in a speed vacuum dryer heated to $50^{\circ} \mathrm{C}$. Next, $200 \mu \mathrm{L}$ buffer A ( $95 \%$ water, $5 \%$ acetonitrile, $0.1 \%$ formic acid) was added, and the digested pellet samples were stored at $-80^{\circ} \mathrm{C}$ without further treatment until required for MS analysis.

\section{LC-MS/MS analysis}

The digested peptide mixtures were pressure-loaded onto a fused silica capillary column

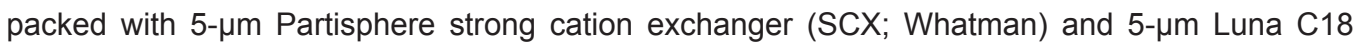


material (RP; Phenomenex), with a Kasil frit at the SCX end. The SCX and RP sections were $2 \mathrm{~cm}$ and $3 \mathrm{~cm}$ long, respectively, and the column was washed with buffer A ( $95 \%$ water, $5 \%$ acetonitrile, $0.1 \%$ formic acid). After desalting, a $9-\mathrm{cm}, 100-\mu \mathrm{m}$ internal diameter capillary with a $5-\mu \mathrm{m}$ pulled tip packed with $5 \mu \mathrm{m}$ Luna C18 material was attached to the two-phase column with a union, and the entire assembly was placed in line with Agilent 1100 quaternary high-performance liquid chromatography (HPLC) equipment for analysis in a 12-step separation. Three buffer solutions were used: $5 \%$ acetonitrile/0.1\% formic acid (buffer A), $80 \%$ acetonitrile/0.1\% formic acid (buffer B), and $500 \mathrm{mM}$ ammonium acetate/5\% acetonitrile/0.1\% formic acid (buffer D). The first step consisted of a 2 -min gradient from $0 \%$ to $15 \%$ buffer $\mathrm{B}$, followed by a 47 -min gradient to $100 \%$ buffer B and 10 min of $100 \%$ buffer B. Next, the buffer was switched to $100 \%$ buffer A in 1 min and kept at $100 \%$ buffer $A$ for another $9 \mathrm{~min}$.

Steps 2-10 had the following profile: $1 \mathrm{~min}$ of $100 \%$ buffer $A, 2 \min$ of $\mathrm{x} \%$ buffer $\mathrm{D}, 5$ min of $100 \%$ buffer $A$, a 2 -min gradient from $0 \%$ to $15 \%$ buffer $B$, a 60 -min gradient from $15 \%$ to $50 \%$ buffer B, a 40 -min gradient from $50 \%$ to $100 \%$ buffer B, and 15 min of $100 \%$ buffer $A$ after a 5-min gradient from $100 \%$ buffer $B$ to $100 \%$ buffer $A$. The 2-min buffer D percentages (x) in steps 2-10 were 5\%, 10\%, 15\%, 20\%, 30\%, 50\%, 60\%, 70\%, and 80\%, respectively. Steps 11 and 12 consisted of $1 \mathrm{~min}$ of $100 \%$ buffer A, 9 min of $100 \%$ buffer D, and 5 min of $100 \%$ buffer A, followed by a 5 -min gradient to $15 \%$ buffer $B$, a 50 -min gradient to $50 \%$ buffer B, a 45 -min gradient to $100 \%$ buffer B, 15 min of $100 \%$ buffer B alone, and 15 min of $100 \%$ buffer A after a 5 -min gradient from $100 \%$ buffer B to $100 \%$ buffer $A$. As peptides were eluted from the microcapillary column, they were electrosprayed directly into a LTQ Orbitrap mass spectrometer (Thermo Fisher Scientific) with the application of a distal $2.5 \mathrm{kV}$ spray voltage. The mass spectrometer was operated in the data-dependent mode. Survey MS scans were acquired in the Orbitrap with the resolution set to a value of 60,000 . Each survey scan $(400-2000 \mathrm{~m} / \mathrm{z})$ was followed by eight data-dependent tandem mass (MS/MS) scans at 35\% normalized collision energy. Automatic gain control target values were 200,000 for the survey scan and 10,000 for the MS/MS scan. Target ions already selected for MS/MS were dynamically excluded for $30 \mathrm{~s}$.

\section{Data analysis}

Tandem mass spectra were searched against the European Bioinformatics Institute International Protein Index mouse protein database using the ProLuCID protein database search algorithm. ProLuCID search results were then filtered with DTA-Select 2.0 using a cutoff of $1 \%$ for false peptide identification rate (-fp 0.01). Peptides with Z score < 4 (i.e., 4 SDs away from the average) or DeltaMass $>7$ ppm (-DM 7) were rejected. Furthermore, the minimum number of peptides to identify a protein was set to 1 (-p 1). Spectral counts for each protein were extracted from DTA-Select files into Excel spreadsheets and normalized to the total spectral counts of the sample from which a protein was identified. Gene ontology analysis was performed using the GSEABase package of BioConductor (http://www.bioconductor.org/). Proteins were divided into biological process, molecular function, and cellular component categories and their subcategories. Expression Analysis Systematic Explorer (EASE) was used to analyze gene ontology and Kyoto Encyclopedia of Genes and Genomes (KEGG) pathways. Genes in a KEGG pathway are judged to be overrepresented if a larger fraction of genes within that pathway is expressed compared with all genes in the genome. GenMAPP was used to analyze gene expression changes, and Cytoscape software was used to draw the picture. 


\section{RESULTS}

In previous studies, the total protein numbers identified in the GV stage, the MII stage, and in the zygotes were 2354, 2973, and 2082, respectively (Wang et al., 2010).

\section{Astacin-like metalloendopeptidase (Astl)}

The astacin family of metalloendopeptidases (EC 3.4.24.21) encompasses a range of proteins found in species from hydra to humans and in mature and developing systems. The functions of Astl include activation of growth factors, degradation of polypeptides, and processing of extracellular proteins (Semenova and Rudenskaia, 2008). The presence of Astl in mouse oocytes has been demonstrated in a previous study (Quesada et al., 2004). However, its role remains unclear. In our study, the expression of Astl1 and Astl2 significantly increased in MII oocytes and decreased when the oocytes developed into zygotes. Given that abundant nutritional stores in oocytes are consumed during the process of development, Astl was suggested as one source of nutrition consumed during the development of oocytes into zygotes. Astacin-like metalloendopeptidase might play an important role in the degradation of maternal proteins and the activation of paternal gene expression.

\section{Selenium-binding proteins}

Twenty selenium (Se)-binding proteins (Txnrd1, Txnrd3, 1300018J18Rik, Gpx1, Gpx3, Prdx2, Sephs1, Sephs2, Sepw1, D5Wsu178e, Txnrd1, Txnrd3, 1300018J18Rik, Gpx1, Gpx3, Prdx2, Sephs1, Sephs2, Sepw1, and D5Wsu178e) were detected in mature mouse oocytes. Se is an essential trace element for humans and animals. It is an antioxidant that prevents free-radical damage and preserves tissue elasticity (Moslemi and Tavanbakhsh, 2011). Se-binding proteins are expressed at different stages. Txnrd1, Gpx4, Gpx1, and Sepw1 are highly expressed during GV or MII stages, but have low expression levels in zygotes. Our findings support the hypothesis that Se is a critical mineral that promotes the development of oocytes and implantation.

\section{Citrate cycle (TCA cycle)}

The citrate cycle, also known as the tricarboxylic acid cycle (TCA cycle), is part of the metabolic pathway involved in the conversion of carbohydrates, fats, and proteins to form energy (Cetica et al., 2003). Mouse oocytes require pyruvate or oxaloacetate as an energy source through the TCA pathway. In our present proteomic data, most of the proteins involved in the TCA cycle were detected. Three TCA proteins (Ogdh, Dld, and Fh1) exhibited high expression in GV oocytes (Figure 1), but the expression of other proteins showed no significant differences. The TCA cycle plays a central role in all living cells because energy production is essential. In eukaryotic cells, the citrate cycle occurs in the matrix of the mitochondria. Therefore, mitochondria are very important organelles for the fertilization of oocytes.

\section{The electron transport chain}

The electron transport chain in mitochondria is the next step following the TCA cycle, and produces adenosine triphosphate (ATP), which is the primary energy source for life. We identified 
26 proteins expressed in oocytes and zygotes, 19 of which exhibited high expression in zygotes. Atp5b and atp5a1, members of the ATP synthase family, exhibited notably high expression, which is beneficial in providing more energy. Transcription of Cox5a and Cox5b was observed in oocytes and during early development (Cui et al., 2006). Cox5a may play an important role in fertilization by selectively interacting with metal ions that are important for fertility and implantation. The electron transport chain utilizes special proteins that can bind metals, and is essential for fertilization because it provides energy for oocytes.

\section{CITRATE CYCLE (TCA CYCLE)}

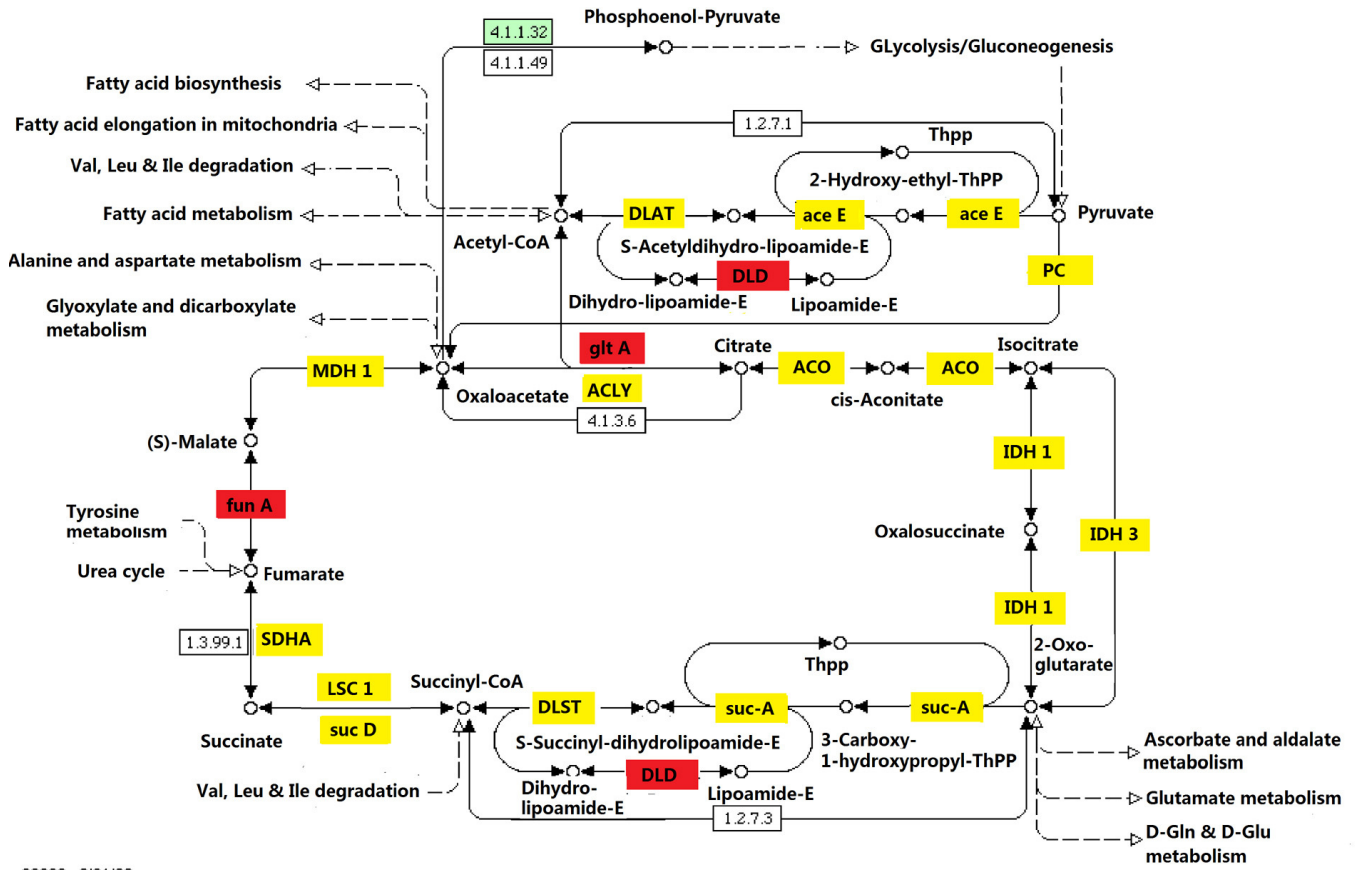

Figure 1. Highly expressed proteins in the citrate cycle (tricarboxylic acid cycle (TCA) cycle). Red rectangles represent proteins expressed more abundantly in germinal vesicle (GV) oocytes compared with metaphase II-arrested (MII) oocytes. Yellow rectangles represent proteins with comparable expression levels in the GV and MII oocytes. Green represents the Kyoto Encyclopedia of Genes and Genomes (KEGG) database.

\section{Urea cycle}

The urea cycle is an essential metabolic pathway that exists in many animals; it plays a key role in removing the toxic metabolite ammonia by converting it to urea and uric acid. We identified seven proteins from the urea cycle in the mouse zygotes: Acy1 (5-peptides), Srm (9-peptides), Oat (10-peptides), Glud1 (19-peptides), Arg2 (21-peptides), Pycrl (21-peptides), and Ckb (683-peptides). It is noteworthy that all seven proteins are only detected in zygotes. Ckb, a 683-amino acid protein in zygotes, is involved in energy homeostasis and reversibly catalyzes the transfer of phosphate between ATP and various phosphagens, such as creatine phosphate. Previous studies have shown that the expression of $\mathrm{Ckb}$ in good-quality embryos is higher than 
in poor-quality embryos (Lee et al., 2010). Ckb may serve as a biomarker or therapeutic target for the developmental potential of oocytes; our present proteomics data confirm that possibility and indicate that the urea cycle plays an irreplaceable role in the development of oocytes to zygotes. This finding suggests that the urea cycle is essential for embryonic development following fertilization.

\section{Fatty acid metabolism}

Oocytes from all mammals contain an endogenous lipid reserve. The involvement of fatty acid metabolism in mouse oocyte maturation is somewhat surprising because mouse oocytes contain only a small amount of stored lipid and readily undergo maturation with simple carbohydrates such as pyruvate as the sole external energy source (Biggers et al., 1967; Eppig, 1976). Therefore, clarification of the regulatory role of fatty acid metabolism in the development of mouse oocytes is necessary. Almost all of the proteins in fatty acid metabolism were detected (Figure 2), but their expression made no significant difference in oocyte development. Although each developmental stage requires fatty acid metabolism, few studies have been conducted to investigate the detailed roles and relevant mechanisms of this metabolism.

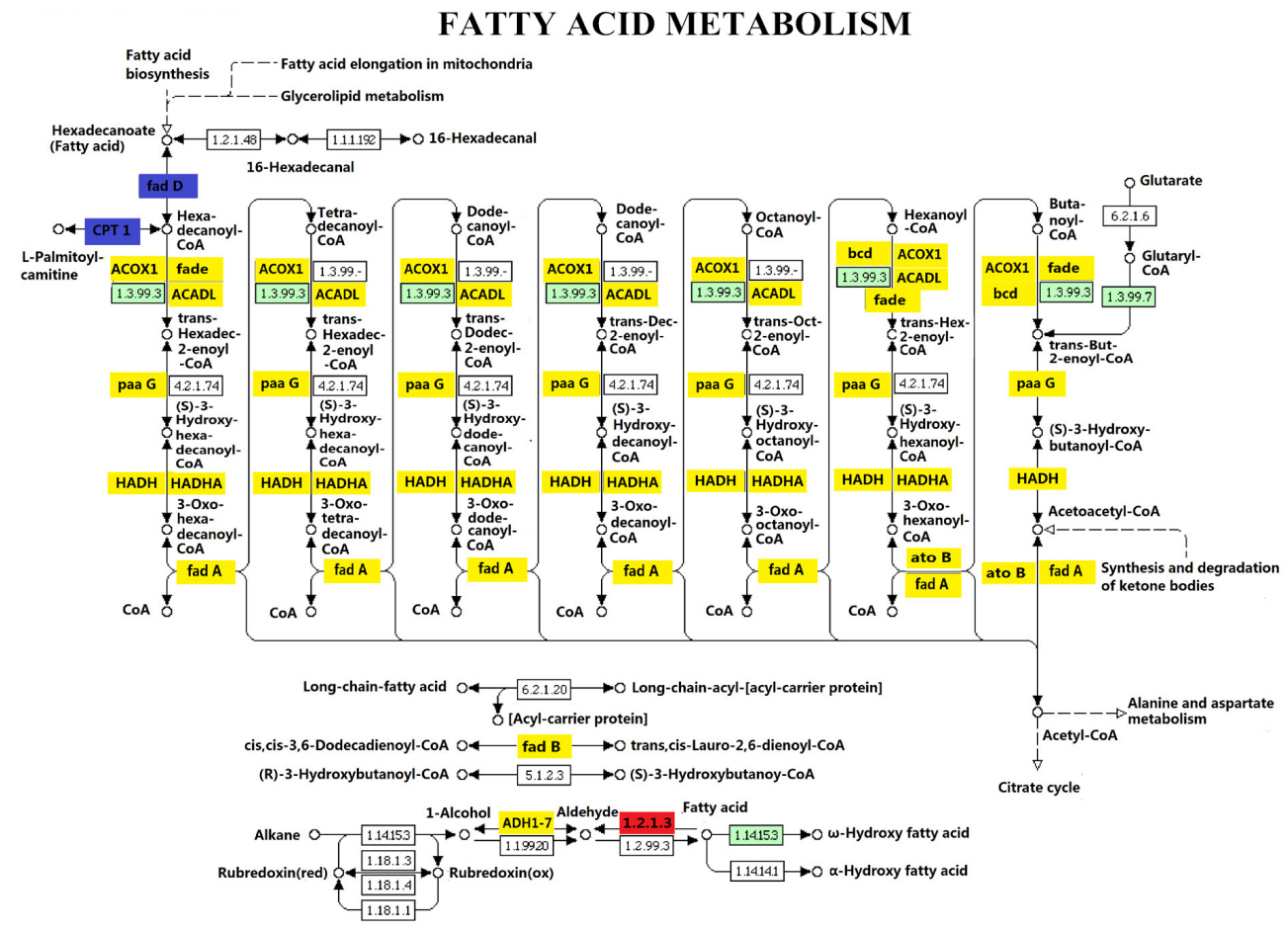

Figure 2. Proteins involved in fatty acid metabolism. Red rectangles represent proteins expressed more abundantly in germinal vesicle (GV) oocytes compared with metaphase II-arrested (MII) oocytes. Blue rectangles represent proteins expressed more abundantly in MII oocytes compared with GV oocytes. Yellow rectangles represent proteins with comparable expression levels in the GV and MII oocytes. Green represents the Kyoto Encyclopedia of Genes and Genomes (KEGG) database. 


\section{Insulin signaling pathway}

Exposure of oocytes to insulin might have a negative impact on the developmental competence of oocytes, which is defined as the ability of oocytes to support embryonic development through the blastocyst stage and/or establish normal pregnancies (Dumesic at al., 2002). Many proteins involved in the insulin signaling pathway were identified in this study; four of them showed elevated expression levels in GV oocytes. In contrast, pyruvate kinase (PYK) was highly expressed in MII oocytes. These proteins may influence the development of oocytes, and our study provides a good foundation for research into the relationship between insulin signaling pathways and oocytes. However, the specific mechanisms underlying this relationship require further study.

\section{DISCUSSION}

Limited information on oocytes and fertilization prevents the efficient therapy of patients with infertility. Additional proteins have been found in the mouse oocyte using new technologies (Wang et al., 2010; Pfeiffer et al., 2011). Nutrition is an essential component for sustaining life, and lack of some nutritional factors might induce fertilization failure or embryonic dysplasia. Therefore, the relationship between nutrition and embryonic development was addressed in the present study.

In contrast to previous methods in which embryo development was compared before and after the addition of one or more nutrients to the culture medium, accurate semiquantitative MS proteomic analysis was employed to detect the content of these nutrients in oocytes and zygotes (Malmström et al., 2009). The results obtained from our recent proteomic studies have provided significant molecular insight into the processes of oocyte maturation, fertilization, and pre-implantation development.

During oogenesis in mammals, the oocyte grows by more than two orders of magnitude, produces large quantities of myriad macromolecules, and undergoes a complex series of morphologic and developmental changes. A tremendous energetic toll is required for the development of oocytes. The oocyte must meet its energetic requirements during development by modulating a number of metabolic pathways that generate ATP. Additionally, nutritional components are essential for sustaining life, and lack of some nutrients might induce fertilization failure or embryonic dysplasia. Therefore, energy pathways such as the TCA cycle and the electron transport chain are crucial for fertilization. Because both pathways occur in the mitochondria, oocytes with low quantities of mitochondria or defective mitochondria may show lower fertilization success. A number of proteins involved in energy metabolism have been detected, and some of these show significant differences in expression levels between oocytes and zygotes. Those proteins that are elevated in MII oocytes may be special factors that are stored in MII oocytes in preparation for the period of fertilization. Fertilization of oocytes requires energy consumption; therefore, metabolic energy production is extremely important. Proteins that are highly expressed in zygotes may be associated with energy production for the development of oocytes.

Based on evidence from previous metabolic labeling experiments and recent proteomic analysis, it seems that the synthesis of these proteins begins during oocyte growth and that the resulting proteins are subsequently stored in the egg for future utilization following fertilization (Tsujii et al., 2004). We found that a number of proteins were highly expressed in MII- or GV-stage oocytes while exhibiting low expression in zygotes, further supporting this hypothesis. In previous studies, Astl has never been associated with embryonic development. In this study, we discovered 
that oocytes stored an abundance of nutrients for further development and that the expression of Astl was 100-fold higher in MII oocytes than in zygotes. These results indicate that Astl may be a crucial factor in early embryo development.

Twelve Se-binding proteins were detected in this study; they are expressed in different stages. Se is an essential element for normal testicular development, spermatogenesis, and spermatozoa motility, indicating its significant role in fertility (Hawkes and Turek, 2001). One previous study showed that Se deficiency can lower reproduction rates in humans and animals (Tang et al., 1991). Previous studies have shown that mouse preimplantation embryos exposed to oxidative stress implant normally when cultured with insulin-like growth factors I and II, epidermal growth factor, insulin, transferrin, and selenium (Meldrum, 1991). Therefore, Se may be critical for the development of oocytes. A Se-rich diet may be helpful for fertilization, which is consistent with traditional Chinese medical science.

In recent years, IVF has become a major treatment method for infertility when other methods of assisted reproductive technologies have failed. Despite recent advances in IVF technology, IVF faces many important challenges that need to be resolved. For example, low implantation rates and high multiple pregnancy rates might lead to our inability to accurately assess the reproductive potential of individual embryos. The development of non-invasive predictors is crucial for overcoming the limitations of morphologic observation as an assessment of the reproductive potential of an embryo. Our recent results indicate the possibility of selecting viable embryos using markers associated with amino acid and carbohydrate metabolism as predictors. In summary, this study provides a novel framework not only for the better understanding of the molecular mechanisms of early embryonic development, but also to achieve quality non-invasive screening of embryos.

We found a number of nutrients involved in the process of oocyte development, revealing the relationship between nutrition and infertility. These data may be helpful for improving in vitro oocyte and embryo culture methods, and for improving women's diets to increase fertilization rates.

\section{Conflicts of interest}

The authors declare no conflict of interest.

\section{ACKNOWLEDGMENTS}

We thank the China Postdoctoral Science Foundation. The specific-pathogen-free-grade hybrid mice \#B6D2F1 (\#C57BL/6 x DBA/2) were housed in the animal facility of the National Institute of Biological Sciences.

\section{REFERENCES}

Biggers JD, Whittingham DG and Donahue RP (1967). The pattern of energy metabolism in the mouse oöcyte and zygote. Proc. Natl. Acad. Sci. USA. 58: 560-567.

Cetica P, Pintos L, Dalvit G and Beconi M (2003). Involvement of enzymes of amino acid metabolism and tricarboxylic acid cycle in bovine oocyte maturation in vitro. Reproduction 126: 753-763.

Cui XS, Li XY, Jeong YJ, Jun JH, et al. (2006). Gene expression of cox5a, 5b, or $6 \mathrm{~b} 1$ and their roles in preimplantation mouse embryos. Biol. Reprod. 74: 601-610.

Dumesic DA, Schramm RD, Peterson E, Paprocki AM, et al. (2002). Impaired developmental competence of oocytes in adult prenatally androgenized female rhesus monkeys undergoing gonadotropin stimulation for in vitro fertilization. J. Clin. Endocrinol. Metab. 87: 1111-1119. 
Eppig JJ (1976). Analysis of mouse oogenesis in vitro. Oocyte isolation and the utilization of exogenous energy sources by growing oocytes. J. Exp. Zool. 198: 375-382.

Gurunath S, Pandian Z, Anderson RA and Bhattacharya S (2011). Defining infertility-a systematic review of prevalence studies. Hum. Reprod. Update. 17: 575-588.

Hawkes WC and Turek PJ (2001). Effects of dietary selenium on sperm motility in healthy men. J. Androl. 22: 764-772.

Lee MS, Liu CH, Lee TH, Wu HM, et al. (2010). Association of creatin kinase B and peroxiredoxin 2 expression with age and embryo quality in cumulus cells. J. Assist. Reprod. Genet. 27: 629-639.

Malmström J, Beck M, Schmidt A, Lange V, et al. (2009). Proteome-wide cellular protein concentrations of the human pathogen Leptospira interrogans. Nature 460: 762-765.

Meldrum DR (1991). Factors affecting embryo implantation after human in vitro fertilization. Am. J. Obstet. Gynecol. 165: 1896-1897.

Moslemi MK and Tavanbakhsh S (2011). Selenium-vitamin E supplementation in infertile men: effects on semen parameters and pregnancy rate. Int. J. Gen. Med. 4: 99-104.

Pfeiffer MJ, Siatkowski M, Paudel Y, Balbach ST, et al. (2011). Proteomic analysis of mouse oocytes reveals 28 candidate factors of the "reprogrammome". J. Proteome Res. 10: 2140-2153.

Quesada V, Sánchez LM, Alvarez J and López-Otín C (2004). Identification and characterization of human and mouse ovastacin: a novel metalloproteinase similar to hatching enzymes from arthropods, birds, amphibians, and fish. J. Biol. Chem. 279: 26627-26634.

Semenova SA and Rudenskaia GN (2008). The astacin family of metalloproteinases. Biomed. Khim. 54: 531-554.

Tang CC, Chen HN and Rui HF (1991). The effects of selenium on gestation, fertility, and offspring in mice. Biol. Trace Elem. Res. 30: 227-231.

Tsujii H, Taniguchi N and Hamano K (2004). Development of Mongolian gerbil embryos in chemically defined media: effects of osmolarity, glucose and phosphate. J. Reprod. Dev. 50: 653-659.

Wang S, Kou Z, Jing Z, Zhang Y, et al. (2010). Proteome of mouse oocytes at different developmental stages. Proc. Natl. Acad. Sci. USA. 107: 17639-17644. 